\title{
MHD Study of Reactor-Relevant High-Beta Regime in the
}

\section{Large Helical Device}

S Sakakibara, K Y Watanabe, Y Suzuki, Y Narushima, S Ohdachi, N Nakajima, F Watanabe, L Garcia $^{1}$, A Weller ${ }^{2}$, K Toi, I Yamada, K Tanaka, T Tokuzawa, K Ida, H Yamada, A Komori, O Motojima and LHD Experimental Group

National Institute for Fusion Science, Toki 509-5292, Japan

${ }^{1}$ Univ. Carlos III, Avda. de la Universidad, 3028911 Leganes, Madrid, Spain

${ }^{2}$ Max-Planck-Institut für Plasmaphysik, IPP-EURATOM-Association, D-17491 Greifswald, Germany

E-mail: sakakibara.satoru@1hd.nifs.ac.jp

\begin{abstract}
In the Large Helical Device (LHD), the volume averaged beta value $<\beta_{\text {dia }}>$ of $5 \%$, which is the highest value in all of heliotron/stellarators and relevant to the reactor requirement, was achieved by optimizing the magnetic configuration from the viewpoint of magneto-hydrodynamic (MHD) characteristics, transport and heating efficiency of the neutral beam. This beta value was instantaneously obtained by pellet injection and maintained for more than $10 \tau_{\mathrm{E}}$, whereas the steady state plasma with a maximum $<\beta_{\text {dia }}>$ of $4.8 \%$ was sustained for $85 \tau_{\mathrm{E}}$ by the gas-puff fueling. While it is predicted theoretically that stochastization of the peripheral magnetic field structure develops with an increment of $\left\langle\beta_{\text {dia }}>\right.$, no serious degradation of the global confinement has been observed in the present $\left\langle\beta_{\text {dia }}>\right.$ range. The several low-order MHD activities located in the periphery were enhanced with the beta value and sometimes affect the local profiles. The amplitude of the mode in the periphery strongly depends on the magnetic Reynolds number, which is close to that of the growth rate and/or the radial mode width of the resistive interchange instability.
\end{abstract}

\section{Introduction}

High beta plasma production is a common subject in magnetic confinement systems for the realization of an efficient fusion reactor. Generally, it is believed that characteristics of MHD equilibrium, stability and transport are key issues for high-beta plasma production in stellarator/heliotrons. Net-current free heliotron plasmas are free from current-driven instabilities unlike in tokamaks, and characterization of pressure-driven instabilities and control of them in the high beta regime are crucial issues for a helical fusion reactor. Since the heliotron has a configuration with weak magnetic shear in the core region and a magnetic hill in periphery, it is predicted that activities of ideal or resistive interchange instabilities and ballooning modes are major key issues for high-beta plasma production. Especially, the understanding of peripheral modes excited by a steep pressure gradient in the magnetic hill region is a common subject in helical devices and tokamaks. Previous Large Helical Device (LHD) experiments indicate that the clear limitation of the beta value due to disruptive phenomena has not been observed in standard operation, whereas several notable phenomena caused by MHD 
instabilities have been observed. In the medium $<\beta_{\mathrm{dia}}>$ range of less than $3 \%$, the variation of plasma profiles with resonant magnetic fluctuations [1] and the minor collapse caused by the formation of a steep pressure gradient in the vicinity of the resonant surface after pellet injection have been observed in the core plasmas, where $\left\langle\beta_{\mathrm{dia}}>\right.$ is the volume averaged beta value estimated by diamagnetic flux measurements and estimated by using the plasma volume and magnetic field strength in vacuum. It is found that these phenomena occur in the marginal region against the low- $n$ ideal interchange mode [2] and the excitation of the mode has not been determined by the Mercier criterion [3]. The observed instabilities are harmless to the global confinement. These MHD activities have not been observed when $\left\langle\beta_{\mathrm{dia}}>\right.$ is increased, which suggests that the resonant surface enters the magnetic well region due to finite-beta effects. MHD modes excited in the periphery with the magnetic hill are enhanced with increasing beta and/or L/H transition [4]. The relationship between the peripheral pressure gradient and the stability boundary against ideal low- $n$ mode has been investigated in the $\left\langle\beta_{\mathrm{dia}}>\right.$ range up to $4 \%$ [5]. Also, in the $<\beta_{\text {dia }}>$ range with more than $3 \%$, peripheral MHD activities become stable spontaneously from the inner region to the outer region when $<\beta_{\mathrm{dia}}>$ exceeds a certain value [6]. This interesting phenomenon has been well observed especially in the $<\beta_{\mathrm{dia}}>$ range of $>4 \%$ [7].

An increase in equilibrium currents with an increment of the beta value leads to a stochastization of the magnetic field structure in addition to an outward shift of the magnetic axis [8], which is likely to reduce the region of plasma confinement. The experiments in Wendelstein 7-AS stellarator for investigating the effect of the stochastization on the plasma confinement suggests that the extension of the stochastic area gave an upper-limit to the pressure gradient in the plasma periphery, which resulted in the limitation of the achieved beta value [9]. Also, the theoretical study of MHD equilibrium for LHD [10] indicates that the area of the "nested" magnetic surfaces is drastically decreased when the beta value exceeds a certain value. The properties of the plasma confinement in the stochastic region have been investigated through the comparison between the connection length of magnetic field lines and the mean free path of the plasma [11]. Also the outward shift of the magnetic axis itself leads to the increase in the effective helical ripple increasing the neoclassical transport. A previous study in the beta range with up to $4 \%$ suggests that the global energy confinement gradually degrades with the beta value, which is caused by the increment of the thermal transport in the periphery [5] and it cannot be interpreted by neoclassical theory because the obtained plasma is in the collisional regime.

In LHD, the production of high beta plasmas has progressed successfully with increasing neutral beam heating power and optimization of the magnetic configuration for heating efficiency, equilibrium and stability, and the highest beta value of $5 \%$ was instantaneously achieved by pellet fueling and the steady state high-beta operation with a maximum $<\beta_{\text {dia }}>$ of $4.8 \%$ was successfully maintained by gas-puff fueling. This article describes the results of recent high beta experiments and the related important physics, MHD equilibrium and stability. An optimization of the magnetic configuration for high-beta plasma production is introduced in the next section. In the third section, after the properties of the obtained high-beta plasma and the behavior of steady-state high-beta 
discharges are described, experimental results on characteristics of MHD equilibrium, stability and its theoretical analysis are shown. Finally, the results are discussed and summarized.

\section{Magnetic Configuration for High Beta Plasma Production}

The LHD is a heliotron device with a pair of continuous helical coils and three pairs of poloidal coils and all of the coils are superconducting. The magnetic axis position $R_{\mathrm{ax}}$ can be changed from 3.4 to $4.1 \mathrm{~m}$ by controlling the poloidal coil currents, changing the characteristics of transport and MHD stability. In the configuration with an inward shift of $R_{\mathrm{ax}}$, there is an improvement in the particle confinement as compared with the outward shifted case because the effective helical ripple loss of neoclassical transport and anomalous transport decrease [12]. In contrast, the inward shifted plasma has a disadvantage with respect to MHD stability because of the magnetic hill formation. The $R_{\text {ax }}$ of $3.6 \mathrm{~m}$ was applied in the high- $\beta$ experiments because highest energy confinement has been obtained and serious MHD activities have not been observed in previous experiments [13].

LHD has another degree of freedom to change the magnetic configuration, namely, the plasma aspect ratio $A_{\mathrm{p}}$. In recent experiments, control of the plasma aspect ratio, $A_{\mathrm{p}}$, was mainly performed in order to optimize the magnetic configuration for high-beta plasma production and investigate MHD characteristics. Since the helical coil (HC) is composed of three layers, $A_{\mathrm{p}}$ can be widely changed by controlling each layer's current [14]. When the central position of the HC current is set inside, the central rotational transform and $A_{\mathrm{p}}$ increases, which reduces the Shafranov shift. High $A_{\mathrm{p}}$ is favorable for heating efficiency and transport because the beta-induced outward shift of the magnetic axis leads to an increase in the helical ripple loss of particles. It is also suitable for raising the equilibrium beta-limit. However, a reduction of the plasma shift restricts spontaneous formation of the magnetic well, and an increment of $A_{p}$ reduces the magnetic shear [15].

The experiments in the configurations with $A_{p}$ of $5.8-8.3$ were done for finding out the optimum $A_{p}$ for high beta plasma production and done under the condition of constant heating power [16]. The maximum beta increases with $A_{p}$ and decreases when the $A_{p}$ exceeds 6.6. While the heating efficiency increased from 0.85 to 0.95 when $A_{p}$ increased from 5.8 to 8.3, edge MHD activity with an $m / n=2 / 3$ spatial structure was enhanced with $A_{p}$, and the minor collapse of the plasma due to the $m / n$ $=1 / 1$ mode occurred in the highest $A_{p}$ configuration where the ideal interchange mode is unstabilized due to weak magnetic shear. The $m$ and $n$ are the poloidal and toroidal mode number, respectively. The heating efficiency is defined as $\left(P_{\text {abs }}-P_{\text {prompt-loss }}\right) / P_{\text {abs }}$, where $P_{\text {abs }}$ is the absorbed power considering the loss of fast ions due to the shine-through effect, and $P_{\text {prompt-loss }}$ is the calculated lost power due to the prompt loss of the fast ions. Thus, recent high-beta experiments have been made in the configuration with $R_{\mathrm{ax}}=3.6 \mathrm{~m}, A_{p}=6.6$ and the toroidal magnetic field at $R_{\mathrm{ax}}, B_{\mathrm{t}}=0.425 \mathrm{~T}$. The optimum $B_{\mathrm{t}}$ was determined experimentally.

\section{Experimental Results}




\subsection{High Beta Discharges}

Figure 1 shows changes of the maximum $<\beta_{\mathrm{dia}}>$ as a function of $\tau_{\text {duration }} / \tau_{\mathrm{E}}$ in the pellet and gas-puff discharges, where $\tau_{\text {duration }}$ is defined as the duration time at which $<\beta_{\text {dia }}>$ with more than $0.9 \times<\beta_{\mathrm{dia}}>\max$ was maintained and $\tau_{\mathrm{E}}$ is the global energy confinement time. The highest- $\beta$ discharge with $<\beta_{\mathrm{dia}}>=5 \%$ was obtained by pellet injection and maintained for more than $10 \tau_{\mathrm{E}}$. The total port-through power of the tangential beams is about $12.3 \mathrm{MW}$. The gas-puff fueling can maintain the discharge during longer time scales. The duration time is determined by the time period of the neutral beam (NB) injection except for the radiation collapse. The discharge with the highest beta value of 4.8 $\%$ using gas-puffing was maintained for $85 \tau_{\mathrm{E}}$. The time development of the discharge is shown in fig.2. The plasma was heated by two co-NB's and a counter NB and the total port-through power is 12.4 MW. The maximum $<\beta_{\mathrm{dia}}>$ of $4.8 \%$ was achieved at $1.29 \mathrm{~s}$ and the ratio of the global energy confinement to the empirical scaling based on International stellarator database [17], $H_{\mathrm{ISS} 95}$, is about 1.1 then. The $<\beta_{\mathrm{dia}}>$ increased with the line averaged electron density, $\bar{n}_{\mathrm{e}}$, and the high-beta plasma was maintained until the NBs were turned off. The gas-puff was applied from $0.6 \mathrm{~s}$ to $1.3 \mathrm{~s}$. The $R_{\mathrm{ax}}$ reached about $3.84 \mathrm{~m}$ at $\left\langle\beta_{\mathrm{dia}}>=4.8 \%\right.$, the corresponding $\Delta / a_{\mathrm{p}}$ is less than 0.25 in the discharge, where $\Delta$ is defined as $\Delta=R_{\mathrm{ax}}-R_{00}$ and $R_{00}$ is the major radius with $m=0$ and $n=0$, and $a_{\mathrm{p}}$ is the effective plasma minor radius, which were calculated by the VMEC code. The Shafranov shift is much smaller than the conventional equilibrium beta-limit defined as $\Delta / a_{\mathrm{p}}=1 / 2$. Peripheral MHD activities such as $m / n=1 / 1$ and $2 / 3$ were dominantly observed in the discharge, and they rotated with $f<10 \mathrm{kHz}$ in the electron diamagnetic direction as shown in fig.2(c). The $m / n=1 / 1$ and $2 / 3$ mode are excited at $\rho$ $\sim 0.9$ and 1 , respectively, where $\rho$ is defined as $\left(\Phi / \Phi_{\text {edge }}\right)^{1 / 2}, \Phi$ and $\Phi_{\text {edge }}$ is the toroidal flux and that surrounded by the plasma edge, respectively. The $m / n=1 / 1$ and $2 / 3$ modes appeared with the increment of $\left\langle\beta_{\mathrm{dia}}\right\rangle$. The appearance of the $m / n=1 / 1$ mode is intermittent compared with that of the $m / n=2 / 3$ mode. The tendency that MHD modes are suppressed from the inner region to the outer one has been well observed in the high-beta regime [6]. The observed peripheral low- $n$ MHD modes are not crucial for the production of high-beta plasmas.

\subsection{MHD equilibrium}

Figure 3 shows a comparison between the plasma profiles observed for the $4.8 \%$ plasma shown in the discharge of Fig.2 and the equilibrium parameters calculated by using the 3-D MHD equilibrium code HINT2 [10]. The connection length of the magnetic field line, $L_{\mathrm{c}}$, for the $4.8 \%$ plasma is shown in Fig.3 (a). The calculation was stopped when the $L_{\mathrm{c}}$ approached $2 \times 10^{3} \mathrm{~m}$. The $L_{\mathrm{c}}$ in the periphery becomes shorter when the $\left\langle\beta_{\text {dia }}>\right.$ increases, corresponding to a reduction of the region of nested magnetic surfaces and to an extension of the stochastic region in the periphery. The volume of the nested magnetic surfaces of the $4.8 \%$ plasma decreases by about $32 \%$ compared to the vacuum case, whereas the stochastic region in the periphery is growing as shown in Fig.3 (d-e). The outer position of the last closed flux surface (LCFS) does not move in spite of the increment of $<\beta_{\text {dia }}>$, while the inner one moves outward. The rotational transform profiles in vacuum and in the $4.8 \%$ case are shown in 
Fig.3 (b). The rotational transform, $t / 2 \pi$, in the periphery seems to be disturbed by finite- $\beta$ effects compared with that in the core region with the nested flux surfaces. Although a magnetic well is formed in the central region by finite-beta effects, the magnetic hill still remains in the periphery. The Mercier criterion, $D_{\mathrm{I}}$, which is well used as the index of the stability boundary of the high- $n$ ideal interchange mode, has a positive value in the periphery, meaning that the ideal high- $n$ mode is unstable. The stability boundary is almost the same as the boundary of the magnetic hill/well. The maximum $D_{\mathrm{I}}$ is about 0.25 at $R=3.35$ and $4.21 \mathrm{~m}$. The stability is discussed in Section 3.3.

The $T_{\mathrm{e}}$ profiles measured at 1.266 and $1.3 \mathrm{~s}$ are shown in Fig.3 (c). While $<\beta_{\mathrm{dia}}>$ and $\bar{n}_{\mathrm{e}}$ are almost the same in both cases, the profiles are slightly different especially in the periphery. For the $T_{\mathrm{e}}$ profile at $1.3 \mathrm{~s}$, the $T_{\mathrm{e}}$ gradients in the regions with $R<3.02 \mathrm{~m}$ and $R>4.48 \mathrm{~m}$ change compared to that in the core region, and the inflection points seem to correspond to the LCFS as shown in fig.3 (d). Also the flattening of the $T_{\mathrm{e}}$ profile appeared around the $t / 2 \pi=3 / 2$ resonant surface and the outer boundary at $T_{\mathrm{e}} \sim 0$ is extended at this time. However, there is no inflection point at $1.266 \mathrm{~s}$ and the flattening is unclear. This phenomenon often has been observed in such high-beta discharges, and there are two possible explanations for this. One is due to the spontaneous change of the static magnetic island formed by finite-beta effects. It has been found out experimentally that the static magnetic island can be changed by local plasma parameters such as collisionality, beta and so on [18]. The magnetic diffusion time is about $12 \mathrm{~ms}$ when the width is assumed as $0.1 \mathrm{~m}$ and $T_{\mathrm{e}}$ is about 0.1 $\mathrm{keV}$, which means that the island width can be changed locally in such short time. Another reason is due to the rotating magnetic island caused by the $m / n=2 / 3$ MHD mode. This mode has been observed even in the low-beta regime and rotates with a saturated amplitude in the discharge as shown in Fig.2. In that case, it can be interpreted that at $1.266 \mathrm{~s}$, no profile flattening appears if the X-point of the $m=$ 2 island is located on the mid-plane at the measured toroidal angle and the O-point makes the flattening at $1.3 \mathrm{~s}$. Profile measurements with faster time resolution are required to investigate whether the magnetic island rotates or not and to distinguish between the stability and equilibrium effects carefully. Thus, a clear limitation of the confinement region and a serious degradation of the plasma confinement due to the stochastization of the magnetic field line has not been observed in the present $<\beta_{\text {dia }}>$ range.

\subsection{MHD Stability}

As shown in fig.3(b), the Mercier mode is unstable in the magnetic hill region. The low- $n$ ideal stability was calculated by using the TERPSICHORE code [19]. In Ref.4, the validity of the low- $n$ stability boundary is discussed through the comparison with the experimentally accessible operational regime, and it suggests that the threshold is $\gamma_{\text {low-n }} / \omega_{\mathrm{A}} \sim 10^{-2}$, where $\gamma_{\text {low-n }}$ is the growth rate of the low- $n$ interchange mode and $\omega_{\mathrm{A}}$ is defined as $\omega_{\mathrm{A}}=v_{\mathrm{A} 0} / R_{0}$, and $v_{\mathrm{A} 0}$ and $R_{0}$ are the Alfvèn frequency and the major radius at the magnetic axis, respectively. In the analysis of the equilibrium shown in Fig.3, no mode with $\gamma / \omega_{\mathrm{A}}>10^{-2}$ was found. The analysis of the high- $n$ ballooning mode indicates that the high- $n$ ballooning mode is unstable in the magnetic hill region with $D_{\mathrm{I}}>0$ [20]. An effect of the ballooning 
mode on plasma profiles and confinement has not been observed so far.

The experiments show that the peripheral MHD activities are dominantly excited with the increase in $\left\langle\beta_{\mathrm{dia}}>\right.$ after the core MHD modes are suppressed by the magnetic well formation due to finite-beta effects [2]. In the periphery, the magnetic hill configuration still remains even if $\left\langle\beta_{\mathrm{dia}}>\right.$ approaches $4.8 \%$ as shown in Fig.3. Here we selected the $m / n=1 / 1$ mode as a representative of the peripheral modes in order to investigate the parameter dependences. The resonance position of the mode is located at $R \sim 3.13 \mathrm{~m}$ and $\sim 4.4 \mathrm{~m}$ when $\left\langle\beta_{\mathrm{dia}}\right\rangle=4.8 \%$, as shown in Fig.3 (b). Figure 4 shows the amplitude of the $m / n=1 / 1$ mode in the $S$ and $<\beta_{\text {dia }}>$ diagram, where $S$ is the magnetic Reynolds number. The data were obtained in the steady-state plasmas in the $\left\langle\beta_{\text {dia }}>\right.$ range of up to $4.8 \%$ and the configuration is the same as that of discharge in the discharge in fig. $2, B_{\mathrm{t}}$ ranging of up to $1.5 \mathrm{~T}$. Closed circles with gray color mean no observation of the $m / n=1 / 1$ mode. In the region with $<\beta_{\text {dia }}><2 \%$ and $S>10^{7}$, no mode was observed or the amplitude was much smaller. The amplitude is enhanced with the increment of $<\beta_{\mathrm{dia}}>$ and with the reduction of $S$, and the $S$ dependence seems to be much stronger than that on $<\beta_{\mathrm{dia}}>$.

Figure 5 shows the changes of the square root of the normalized mode amplitude of the $\mathrm{m} / \mathrm{n}$ $=1 / 1$ mode, $\left(\tilde{b}_{\theta} / B_{\mathrm{t}}\right)^{1 / 2}$, as a function of $S$ in the $<\beta_{\text {dia }}>$ range of $2.5-3.0 \%$ and $\left|I_{\mathrm{p}} / B_{\mathrm{t}}\right|<20 \mathrm{kA} / \mathrm{T}$, where $I_{\mathrm{p}}$ is the net toroidal current. Here the island width, which is proportional to $\left(\tilde{b}_{\theta} / B_{\mathrm{t}}\right)^{1 / 2}$, is considered in order to compare with the $S$ dependence of the radial mode width of the resistive interchange mode. The envelope of $\left(\tilde{b}_{\theta} / B_{\mathrm{t}}\right)^{1 / 2}$ decreases with $S$ and is proportional to $S^{-0.355}$. Figure 6 shows the changes of the growth rate of the resistive interchange mode, $\gamma_{\mathrm{r}}$, normalized by $\omega_{\mathrm{A}}$ and the normalized mode width, $\delta / a_{\mathrm{p}}$, as a function of the logarithm of $S$ in the different $D_{\text {II }}$ plasmas, resulting from calculations with the FAR3D code [21, 22]. The $\delta$ is the half width of the radial structure of the $m / n=1 / 1$ mode. To quantitatively investigate the tendency, the pressure profile is fixed as $P=P_{0}\left(1-\rho^{2}\right)\left(1 .-\rho^{8}\right)$ and $D_{\mathrm{I}}$ is changed by increasing $\beta_{0}$. The $D_{\mathrm{I}}$ changes from -0.05 to 0.44 when the $\beta_{0}$ increases from $1.5 \%$ to $5.6 \%$. While the ratio $\gamma_{\mathrm{r}} / \omega_{\mathrm{A}}$ increases with beta, it decreases with the increment of $S$ for any beta case. The $\gamma_{\mathrm{r}} / \omega_{\mathrm{A}}$ is proportional to $S^{-1 / 3}$ for $D_{\mathrm{II}}<0$. When $D_{\mathrm{I}}>0, \gamma_{\mathrm{r}} / \omega_{\mathrm{A}}$ approaches the growth rate of the ideal mode with the increment of $S$. The $S$ dependence in fig. 5 was obtained in the ideally-stable plasmas and in the $S$ range with $7 \times 10^{5} \sim 2 \times 10^{7}$, meaning that the range of the validity of the relationship of $\gamma_{\mathrm{r}} / \omega_{\mathrm{A}} \propto S^{-1 / 3}$ is the same as in the theoretical calculation. The mode width is deeply related to $\gamma_{\mathrm{r}} / \omega_{\mathrm{A}}$, and it is also proportional to $S^{-1 / 3}$. Thus, the $S$ dependence of the amplitude of the mode is close to that of the linear growth rate and the radial mode width of the resistive interchange instability.

\section{Discussion and summary}

A high- $\beta$ plasma of $5 \%$ was successfully obtained through the $A_{\mathrm{p}}$-scan experiments and the increase in heating power, and a steady-state high-beta plasma with a maximum $<\beta_{\mathrm{dia}}>$ of $4.8 \%$ was maintained for a much longer time than the energy confinement time. A previous study shows that the $H_{\mathrm{ISS} 95}$ gradually degrades from 1.6 to $1.0-1.2$ when $<\beta_{\mathrm{dia}}>$ increases. The transport analysis indicates that the thermal transport in the periphery significantly degrades with an increment of $<\beta_{\mathrm{dia}}>$, and the transport 
coefficient in the high-beta regime is about ten times larger than the prediction by the gyro-reduced Bohm model [23]. As one of the reasons for the degradation, turbulence caused by the resistive-g mode has been considered. The predicted transport coefficient in the periphery is quantitatively consistent with experimental results. The turbulence is mainly caused by the decrease of $S$ and the increment of the pressure gradient. Thus, reduction of the transport due to turbulence is expected in high- $S$ plasmas. Also the effect of the high- $n$ ballooning mode that is expected to be unstable in the periphery with the magnetic hill should be clarified.

It is predicted that the stochastization of the magnetic field structure will become a major subject in plasmas with higher-beta values. Since LHD has dense rational surfaces in the periphery, the overlapping of the magnetic islands easily occurs due to the equilibrium current, ergodizing the magnetic field line. The effect on plasma confinement has not been clear because the MHD activity still remains in the periphery. However, a clear limitation of the confinement region and a serious degradation of the plasma confinement have not been observed in the present beta range. One of the reasons that the pressure gradient in such stochastic regions is maintained, may be that the perpendicular transport is dominant rather than the parallel one in such collisional high-beta plasmas. Therefore, it is important to clarify the dependence of the plasma confinement on the collisionality. In the high-beta discharges, the Shafranov shift is much smaller than that given as the equilibrium beta-limit. However, it still decreases the heating efficiency. Active control of the magnetic axis is planned and expected to contribute to maintaining a high heating efficiency.

A clear $S$ dependence of the amplitudes of MHD modes has been found in ideally stable plasmas, and it is almost the same as that of the linear growth rate and mode width of the resistive interchange mode. In the plasma with the $<\beta_{\mathrm{dia}}>$ of $4.8 \%, D_{\mathrm{II}}$ around the $1 / 2 \pi=1$ resonance is about 0.1 , meaning that the $S$ dependence of the mode is still maintained. The onset of the resistive interchange mode is in agreement with the linear theory of the resistive mode [24], whereas the relationship between the non-linear saturation level and $S$ is theoretically unclear yet. The experimental facts suggest that at least, low- $n$ MHD activity in the magnetic hill becomes more stable in the high- $S$ plasma, which is favourable for a fusion reactor. However, low- $n$ ideal modes having a growth rate (or radial mode width) exceeding a certain limit should be avoided. The understanding of these results is essential for considerations of techniques of mode stabilization.

\section{Acknowledgement}

This work is supported by NIFS under contract No.NIFS05ULPP534 and the Ministry of Education, Science, Sports and Culture, Grant-in-Aid for Scientific Research (B), 19360419, 2007.

\section{References}

[1] Sakakibara S et al 2001 Nucl. Fusion 411177

[2] Sakakibara S et al 2002 Plasma Phys. Control. Fusion 44 A217

[3] Yamada H et al 2001 Plasma Phys. Control. Fusion 43 A55

[4] Toi K et al 2005 Phys. Plasmas 12020701 
[5] Watanabe K Y et al 2005 Nucl. Fusion 451247

[6] Komori A et al 2005 Phys. Plasmas 4512056122.

[7] Sakakibara S et al 2006 Proc. 21st Int. Conf. on Fusion Energy 2006 (Chengdu, 2006) (Vienna: IAEA) CD-ROM EX/7-5

[8] Yamada H et al 1992 Nucl. Fusion 3225

[9] Zarnstorff M C et al 2004 Proc. 20th Int. Conf. on Fusion Energy 2004 (Vilamoura, 2004)

(Vienna: IAEA) CD-ROM EX/3-4

[10] Suzuki Y et al 2007 Proc. Joint Conf. 17th Int. Toki Conf. And $16^{\text {th }}$ Int. Stellarator/Heliotron Workshop(Toki, 2007) CD-ROM P2-043

[11] Watanabe K Y et al 2007 Plasma Phys. Control. Fusion 49605

[12] Yamada H et al 2005 Nucl. Fusion 451684

[13] Yamada H et al 2002 Plasma Phys. Control. Fusion 44 A245

[14] Motojima O 1995 Fusion Technol. 27123

[15] Ichiguchi K et al 1996 Nucl. Fusion 361145

[16] Sakakibara S et al 2006 Fusion Sci. Technol. 502177

[17] Stroth U et al 1996 Nucl. Fusion 361063

[18] Narushima Y et al accepted to Nucl. Fusion

[19] Cooper W A 1992 Plasma Phys. Control. Fusion 341011

[20] Nakajima N et al 2007 Proc. Joint Conf. 17th Int. Toki Conf. And $16^{\text {th }}$ Int. Stellarator/Heliotron Workshop(Toki, 2007) CD-ROM P1-048

[21] Garcia L 1988 Proc. 25th EPS Int. Conf. (Prague, 1998) 22A, Part II (1998) 1757

[22] Matsumoto T et al 2007 Proc. Joint Conf. 17th Int. Toki Conf. And $16^{\text {th }}$ Int. Stellarator/Heliotron Workshop(Toki, 2007) CD-ROM P1-043

[23] Funaba H et al 2007 Fusion Sci. Technol. 511129

[24] Sakakibara S et al 2006 Plasma Fusion Res. 1049 


\section{Figure Captions}

\section{Figure 1}

Changes of the maximum averaged beta value as a function of the duration time where $\left\langle\beta_{\mathrm{dia}}\right\rangle>$ $0.9 \times<\beta_{\text {dia }}>{ }_{\text {max }}$.

\section{Figure 2}

Time development of (a) $<\beta_{\mathrm{dia}}>, \bar{n}_{\mathrm{e}}$, injection of neutral beam, (b) $R_{\mathrm{ax}}$, (c) frequencies of $m / n=1 / 1$ and $2 / 3$ modes, and amplitudes of (d) $m / n=1 / 1$ and (e) $2 / 3$ modes in high-beta discharge with $4.8 \%$.

\section{Figure 3}

Radial profiles of (a) $L_{\mathrm{c}}$, (b) $\mathrm{v} / 2 \pi$ at $<\beta_{\mathrm{dia}}>=0$ and $4.8 \%$, (c) $T_{\mathrm{e}}$ profiles at $\mathrm{t}=1.266$ and $1.3 \mathrm{~s}$ and puncture maps of magnetic field lines at (d) $<\beta_{\mathrm{dia}}>=4.8 \%$ and (e) $0 \%$.

\section{Figure 4}

Amplitude of the $m / n=1 / 1$ mode in the $S$ and $<\beta_{\text {dia }}>$ diagram.

\section{Figure 5}

Changes of square root of the amplitude of the $m / n=1 / 1$ mode as a function of $S$.

Figure 6

Changes of (a) normalized growth rate and (b) normalized radial mode width of resistive interchange mode as a function of the logarithm of $S$ in different $D_{\text {I }}$ plasmas. 


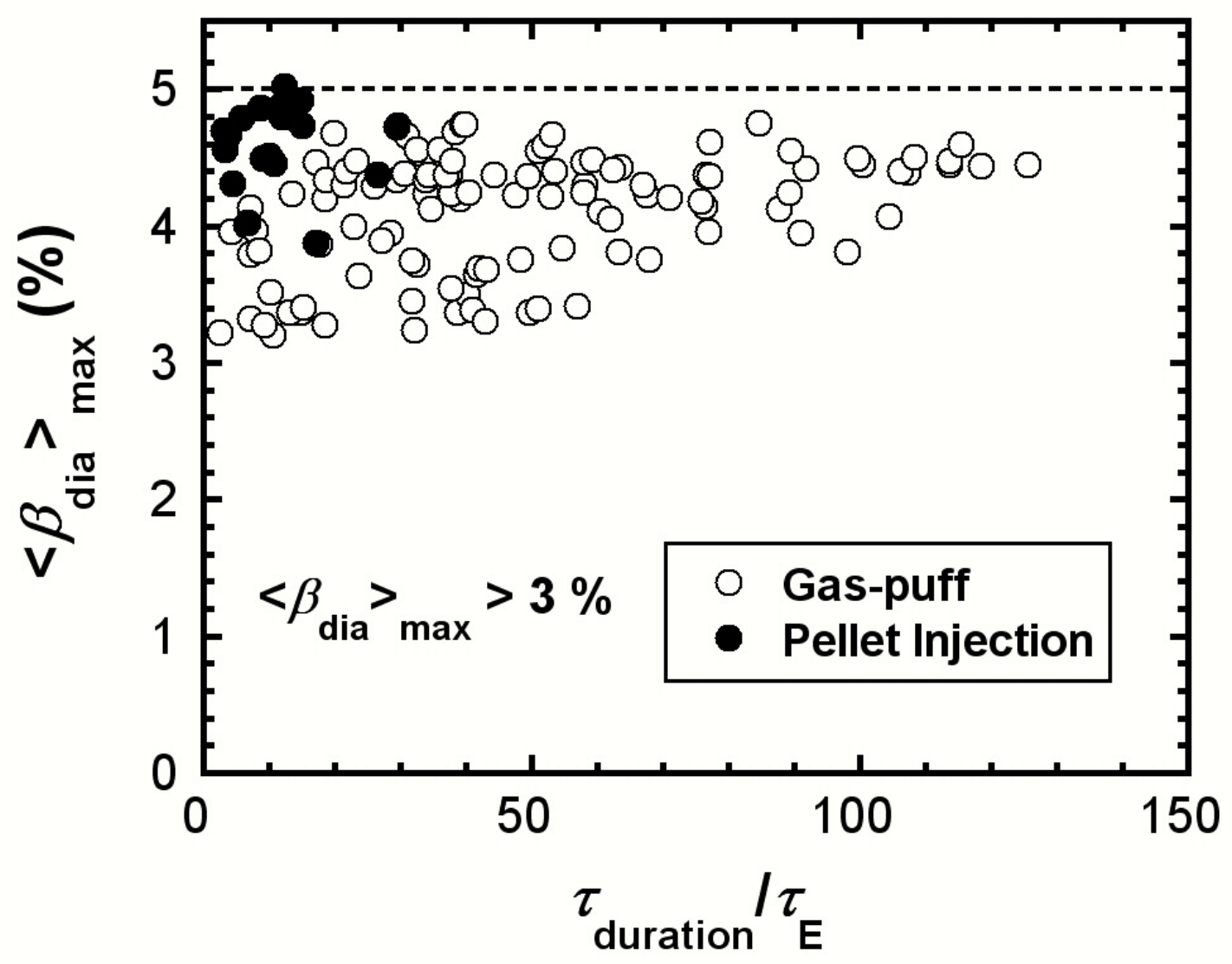

Figure 1 


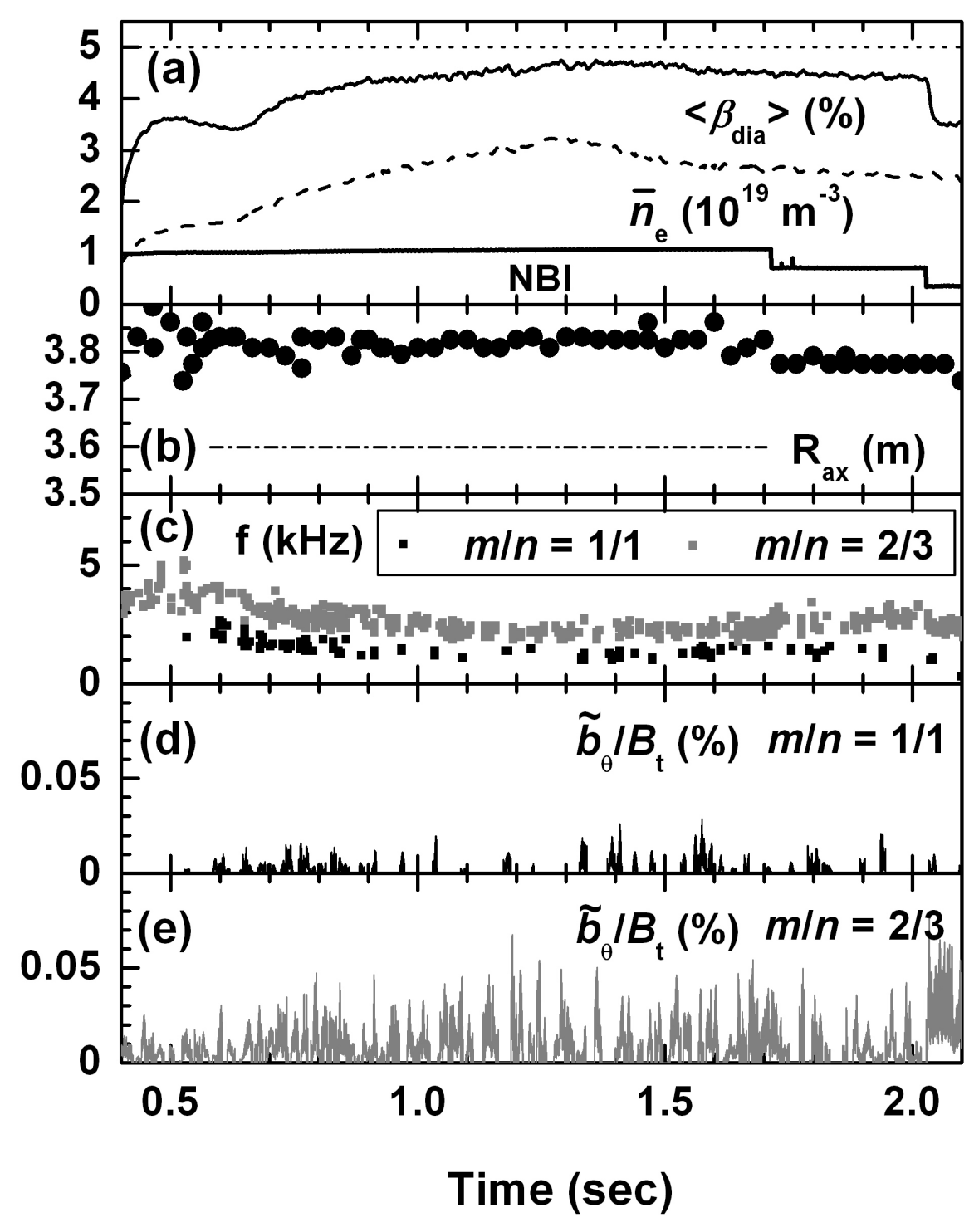

Figure 2 


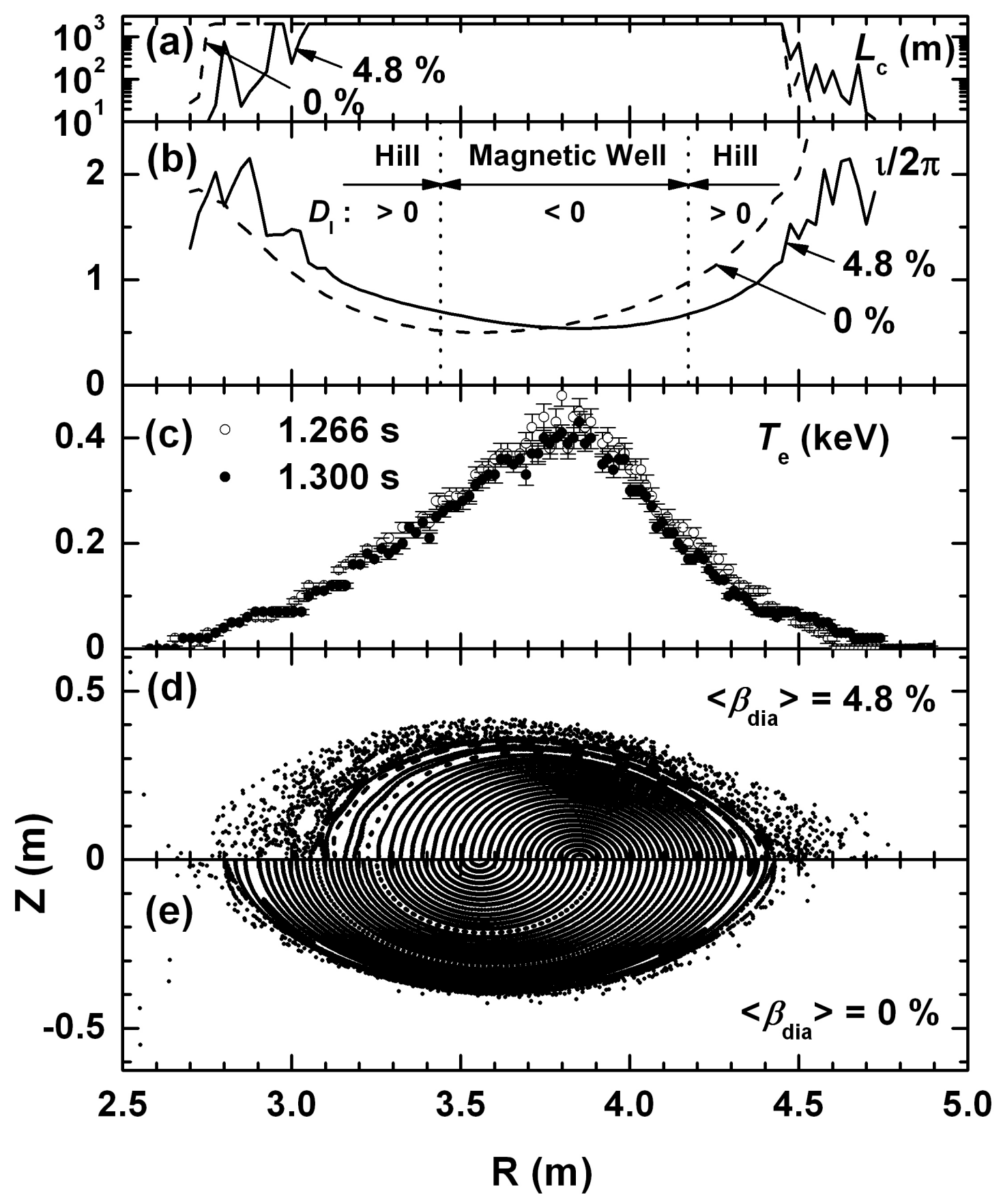

Figure 3 


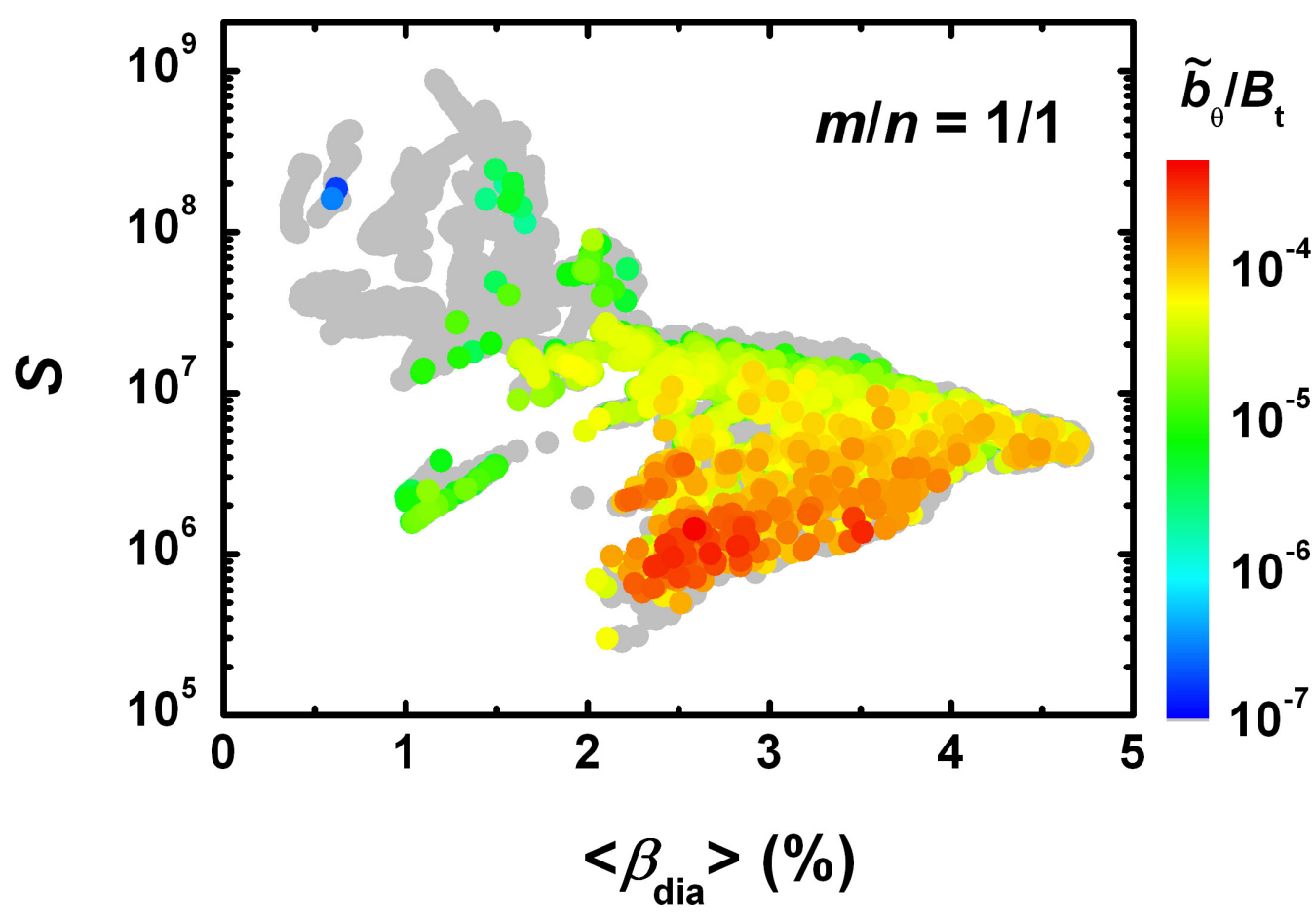

Figure 4 


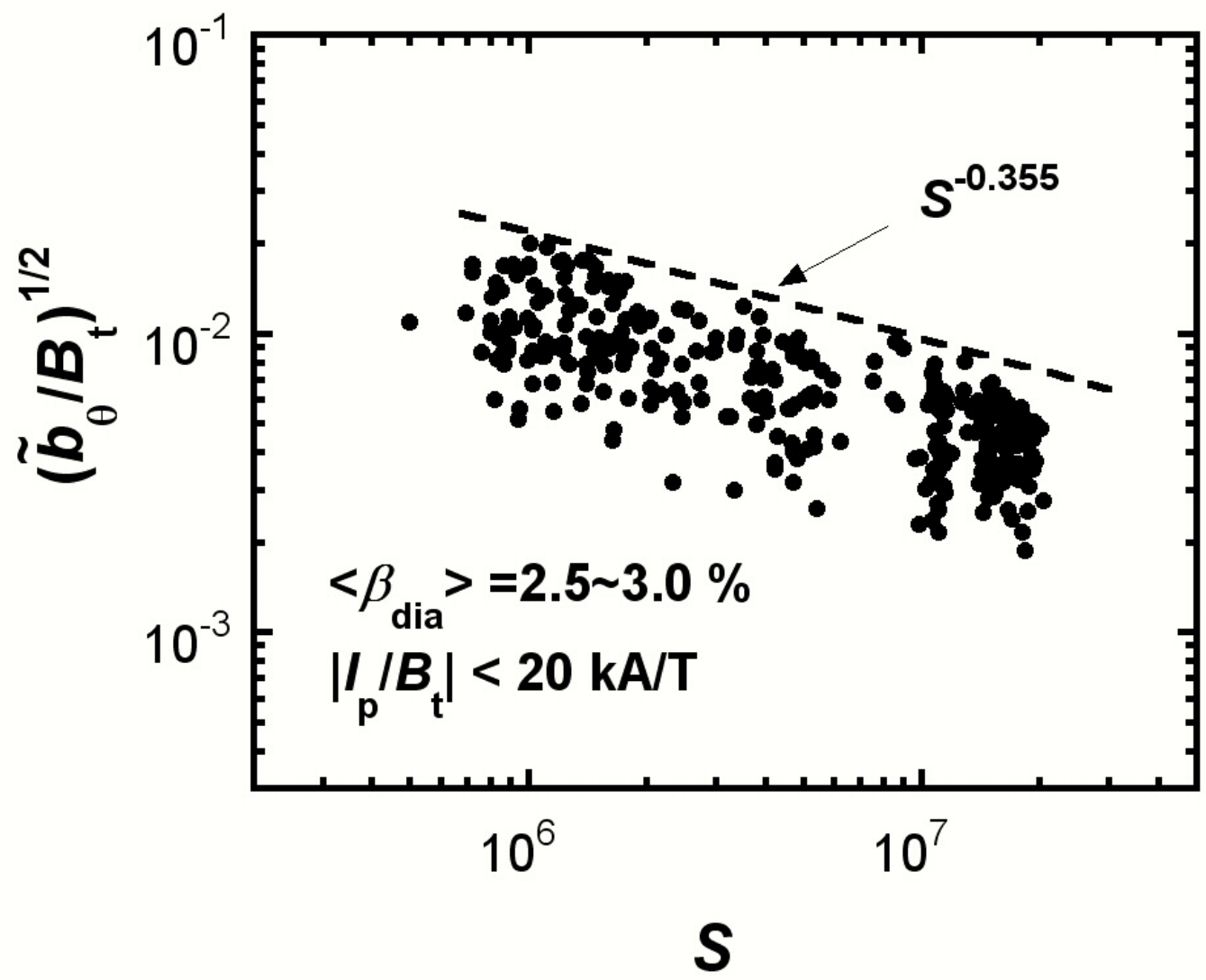

Figure 5 


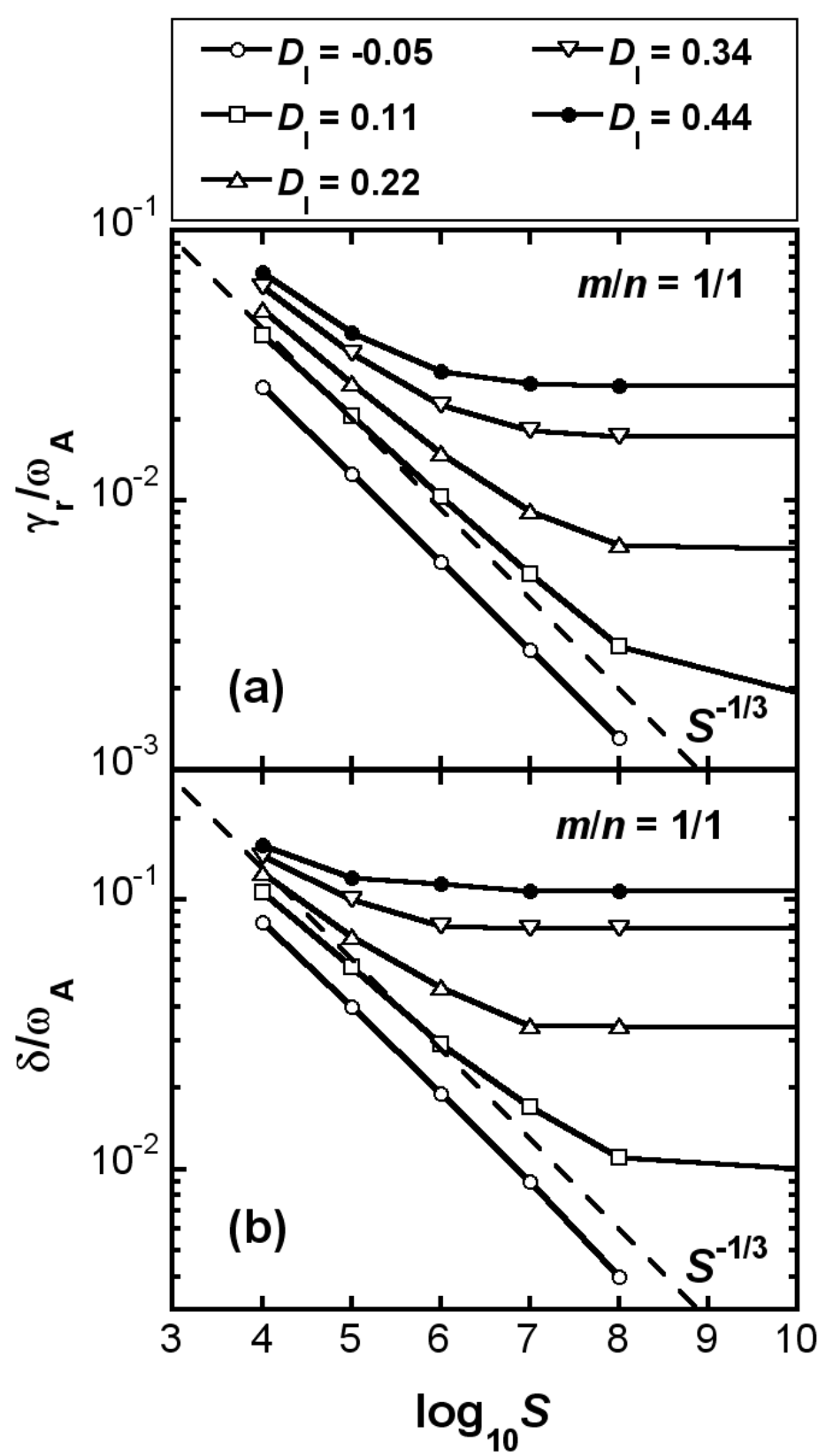

Figure 6 